\title{
Performance Testing Program Design and Assessment Methods of Small Arms
}

\author{
Zhang Junbin, Huang Xueying, Ma Li, Xu Youliang, Li Hongkui \\ China Baicheng Ordnance Test Center
}

Keywords: Small arms, operational performance test, environment simulation.

\begin{abstract}
In this paper, a comprehensive identification on the overall performance of light weapons is as a starting point, and it proposed simulated combat background, operational performance testing program of light weapons with a typical combat mission, and to build simulated battlefield environment and simulate human testing apparatus to establish methods are discussed, proposed the construction methods and technical approach. On the basis of analysis of the various performance evaluation methods, mathematical model of light weapons and tactical performance test evaluation is established.
\end{abstract}

\section{Introduction}

Weapons and equipment for combat use, the merits of using performance is evaluated as an important dimension of weaponry, how to ensure that weapons tests through stereotypes, "easy to use, effective, and durable", and "able to fight, to win the war" requirement is equipped to work under the new situation urgent need. As departments assessment of weaponry, the state should provide a comprehensive range test performance, simple environment to test mode transition is a complex test environment to tactical performance, individual performance by the technical performance, conduct tests to identify weapons systems under near-real conditions, weapons systems to combat the use of performance make the right objective, scientific evaluation.

\section{Simulated Battlefield Environment Construction Method}

According light weapons combat characteristics, combat and weapons requirements, light weapons and equipment during use may experience environmental conditions mainly topography and climate, situational awareness and communications networking environment, electromagnetic environment, against the environment, sound and light smoke environment, therefore, to build simulation battlefield environment must be considered from the following aspects:

(1) The topography and climate conditions Build

Through the use of light weapons and combat environmental analysis, you can get weapons and equipment during use may experience a variety of terrain and climatic conditions, topography geographical attributes, vegetation, structure, shape, geology, and climate parameters of temperature, humidity, barometric pressure, wind, rain, snow, fog, dust and other statistical parameters, it is possible to obtain the topography of light weapons and climatic conditions in the real data, the use of mathematical methods for processing, the establishment of relevant databases.

Way to build terrain using built environment, field area to simulate typical geographical environment, such as sand, grass, ditches, rice paddies, the walls, single-plank bridge, rail, barbed wire, etc; typical climate simulation using analog equipment such as wind, rain, sand dust and so on. (2) Construction of situational awareness and communications networking environment

Sensor Networks light weapons systems and communications networking network situational awareness by "seamless" connections against network and communications networks. Sensor network through the sensor case is passed to the target command and control centers, information will be processed after the command and control center passed to the fire units, fire units will hit the target information is passed to the post command and control center and sensors, to achieve understanding of spatial interaction. 
According to the characteristics of light weapons in combat, battlefield situational awareness of the need to build semi-physical simulation methods and communications networking, use of manradio, monitor radio, man computer, software and other equipment for combat command simulations, using real equipment and a combination of analog devices way construction group network communication, information transfer test environment.

(3) Against the Environmental Construction

Under range conditions, light weapons and tactical performance test can not take the form of mounting confrontation were, from safety considerations, the use of target simulation target simulated enemy personnel, target simulation target mainly by explicit and implicit target, moving explicit and implicit target and light armored targets, to simulate a single target (chest target, half of the target, the body of the target), the cluster target, running goals. Each target is a separate unit with its own number is controlled, the control after the control commands received by the radio system, the information is transmitted to the control circuit of the processor, after the number is completed, the target work. When the projectile hits the target, hit the sensor energy projectile impacting target information to the control circuit processor, when the hit information is correct, the target began to play down accordingly.

(4) Construction of the electromagnetic environment

Electromagnetic environment, including natural and man-made electromagnetic environment electromagnetic environment, complex electromagnetic environment is a form of electromagnetic environment in the time domain, frequency domain, airspace, to a domain, which mainly consists of electronic warfare environment, radar environment, communication environment, photoelectric environment like. Infantry units is a relatively large mobility platform may encounter electromagnetic environment is very complex, so as to soldiers in armored vehicles, aircraft or ships, communications equipment and other command electromagnetic environment as a basic condition to determine which man electromagnetic environmental conditions, the use of electromagnetic signals analog Devices analog electromagnetic environment.

(5) The sound and light smoke Environmental Construction

Light weapons in actual combat experience gunfire, officers cries, fire, smoke and other environments. According to the battlefield environmental standards, determine the sound, fire and smoke as the main simulation object. Use battlefield smoke generator, battlefield fire generating device, audible sound and light smoke simulation device emulation environment that can simulate the sound and light smoke effect to the provisions stipulated in the geographic area and time specified.

In the range test conditions, the building can only be simulated battlefield environment is a typical environment, the natural environment to simulate typical light weapons in the actual use of the experience and some induced environment, and comprehensive operational environment simulation, the need to establish an alternative to the real environment virtual combat environment, not only can quantitatively describe the various features of the operational environment and behavior, but also that the scientific development of test programs to optimize test methods to verify the scientific and operational, personnel training and test evaluation test programs provide the means

\section{Light Weapon Performance Testing Program Design}

\section{Test overall program}

Light weapons should be close to the actual battlefield performance testing requirements to achieve systematic, operational function, information interoperability, networking firepower, combined effectiveness of identification, test the overall program design from the following three aspects:

(1) Analysis to determine the weapon system using a cross-sectional view, through the strike targets at different distances, assessment weapon system for precision strike capability and damage the effectiveness of different objectives;

(2) By setting the typical situational awareness and communications networking environment, assessment information transfer weapons systems, interoperability, situational awareness, graphic 
interpretation, group network communication capabilities;

(3) through the development of the tactical team would like to be, construction typical topography and target simulation system, according to the team attack, defense, urban warfare, combat encounters and other typical usage patterns, weapons into the system, personnel into the establishment, assessment-man team to use weapons system networking firepower capability and performance under simulated battlefield tactical use of background conditions.

\section{Test Mode}

According to the purpose of combat operations, the nature of its manifestations, combined with the main task of the future may be responsible for the infantry units, light weapons and operational performance testing required by the offensive combat, defensive battle, fighting and urban combat encounter four modes, according to the typical form of combat, develop tactical scenario, carry out performance testing under typical environmental conditions of the battlefield, the test requirements are as follows:

a. Test environment similar to actual combat, realistic battlefield environment;

b. Number shooter several classes, height, weight, age, fitness and education level, etc. should be representative;

c. Shooter shooting stance taken as standing, kneeling, prone, travel, leap, roll and other combat firing mode;

d. Against the environment for a variety of man-goal target. Target number is the number one enemy classes or perform a typical combat mission platoon encountered. Distance between the target and the looming timing are unknown, and simulate actual combat enemy targets as possible.

\section{Assessment Methods}

Complete the required task light weapons in combat ability of the need to use whether you can use at any time, whether the use of reliable, if there is sufficient capacity to evaluate three aspects. Weapon system performance evaluation methods are shooting efficiency evaluation method, ADC, fuzzy comprehensive evaluation method, AHP, expert scoring method. From the perspective of a comprehensive assessment system derived mathematical equations integrated assessment of operational performance, the mathematical model of light weapons and combat performance integrated assessment.

Light weapons systems and tactical performance of the comprehensive assessment, the mathematical essence is multi-objective optimization problem by seeking optimal multi-objective function to obtain the overall performance of the system. In obtaining the various performance indicators weapon system, based on the performance parameters of the weapon system and related factors attributed to a number of mathematical models to reflect the relationship and specific algorithms to calculate the overall effectiveness of weapon systems [2.8].

For weapons systems, always want bigger and better overall system performance, according to the characteristics of light weapons combat test, build a multi-objective function equation (1) to obtain the maximum weapon system performance.

$$
\begin{gathered}
(V P)\left\{\begin{array}{l}
V-\max _{x \in R} F(x) \\
R=\{R / G(x) \geq 0\}
\end{array}\right. \\
F(x)=\left[f_{1}(x), f_{2}(x), \cdots \cdots f_{n}(x)\right]^{T} \\
R=\{R / G(x) \geq 0\} \\
S=F(x) \\
x=\left[\begin{array}{lll}
x & x & \mathrm{~L} \quad x_{n}
\end{array}\right]^{T} \in R \\
G(x)=\left[\begin{array}{l}
g_{1}(x), g_{2}(x), \cdots \cdots g_{n}(x)
\end{array}\right]^{T}
\end{gathered}
$$

Among them:

In the formula

$F_{X}-$ Overall efficiency of the system; 
$f(x)$ _- Comprehensive performance parameters;

$R-$ Feasibility solution set of decision space, that solution set of performance indicators;

$g_{i}(x)$ __Experimental estimates;

$S$ - Target space feasible point sets;

$x$ - Decision vector, ie individual performance parameters;

$G(x)$ _ Constraint function vector;

Equation (1) is a vector constraint extremum problem by comparing the pros and cons, to seek the maximum value of the overall effectiveness of the weapon system. In order to obtain the maximum weapon system performance, it must be the performance parameters of each individual weapon systems combined with the comprehensive performance parameters, obtained as a function of overall performance and performance parameters [3,4.9]. That set up the following equations:

$$
\left.\begin{array}{l}
E=\left\{e_{1}, e_{2}, \cdots \cdots e_{n}\right\} \\
e_{i}=f\left(x_{1}, x_{2}, \cdots \cdots x_{n}\right)
\end{array}\right\}
$$

In the formula

$$
\text { E-Overall Effectiveness; }
$$

$e_{i}$ - Quality factor;

Due to the different dimensions of various performance parameters of light weapons systems, can not be unified metrics and weapon system performance parameters require some bigger and better, and some require the smaller the better, so that the overall effectiveness of the system in order to obtain the greatest weapon Solutions value must be standardized [5,6] for various performance parameters, standardized linear variation method can be used:

$$
V_{i}(x) \begin{cases}1 & f_{i}(x) \geq f_{i}^{*} \\ \frac{f_{i}(x)-f_{i}^{0}}{f_{i}^{*}-f_{i}^{0}} & f_{i}^{0} \prec f_{i}(x) \prec f_{i}^{*} \\ 0 & f_{i}(x) \leq f_{i}\end{cases}
$$

In this formula

$f_{i}^{*}$ - The optimum value of $f_{i} x$;

$f_{i}^{0}$ - The worst value of $f_{i} X$;

$V_{i}(x) \ldots$ Standardization of the objective function.

After each performance index system standardization, should clear the importance of various performance indicators, namely the various performance parameters on the overall effectiveness of the contribution - contribution factor, given in the form directly to quantify the various performance parameters important in the overall performance of degree. The method can be obtained by weighted performance parameters on the overall performance of each contribution factor, by weighing the various performance parameters, evaluation, to achieve the purpose balanced, so that the overall weapon system performance can be obtained optimal function:

$$
W_{T}=W\left(\omega_{1}, \omega_{2}, \cdots \cdots \omega_{i}\right)
$$

In this formula $W_{i}$ - The overall effectiveness of the weapon system function;

$\omega_{i}$ - Contribution Factor。

Comprehensive formula (1), (2), (3), (4), you can get a light weapon system performance evaluation method for mathematical expressions:

$$
(V P) \quad\left\{\begin{array}{l}
\max _{x \in R} F(x)=\left[V_{1}(x), V_{2}\left(x \cdots \cdots V_{i}(x)\right)\right]^{T} \\
R=\left\{x / G(x)=\left[g_{1}(x), g_{2}(x), \cdots \cdots g_{n}(x)\right]^{T}\right\} \\
x=\left[\omega_{1}, \omega_{2} \cdots \cdots \omega_{n}\right]^{T}
\end{array}\right.
$$

The use of the simplex method can solve for equations (5) to give: 


$$
F\left(\bar{x}^{(1)}\right)=\left[\omega_{1} \bar{x}^{(1)}, \omega_{2} \bar{x}^{(1)} \cdots \cdots \omega_{m} \bar{x}^{(1)}\right]^{T}
$$

Requirements and performance values are compared,

$$
F^{0}=\left[f_{1}^{1}, f_{2}^{2}, \cdots \cdots f_{m}^{m}\right]^{T}=\left[c_{1} x^{1}, c_{2} x^{2}, \cdots \cdots c_{m} x^{m}\right]^{T}
$$

Obtain a difference between the two

$$
\nabla=\left|F\left(\bar{x}^{(1)}\right)-F^{0}\right|
$$

$\nabla_{\text {is }}$ The smaller the better, so iterations continue until satisfied.

Weapon System Operational Effectiveness Analysis methods varied, can be summed up into three categories: statistics, analytical method and combat simulation. Currently, the Lanchester Equation and computer war simulation combines a variety of marine models posed in terms of weaponry and tactical effectiveness evaluation has been widely applied. In Lanchester first linear law as the basis, considering the probability of finding weapons systems, destroying probability, decision time, the fire attack strength, can create dynamic variable structure wartime differential equations, allowing for light weapons and combat effectiveness evaluation.

\section{Conclusion}

In this paper, light weapons and operational performance testing program design and assessment methods are discussed, as the following conclusions:

1. Determine the simulated combat background, light weapons operational performance testing method with a typical combat mission.

2. Propose construction method of simulated battlefield environment and human testing apparatus and technical approach.

3. Put up The mathematical model and method of light weapons and tactical performance test evaluation.

This paper proposed a method to build a battlefield environment, testing programs and assessment methods to carry light weapons and combat performance tests is of guiding significance.

\section{References}

[1] Bao jiandong Man - Gun experimental study of the interaction, 2006.

[2] Xiong daguo, "Stochastic process theory and application, Beijing: National Defence Industry Press, 1991.

[3] Zhao wei, "Stochastic Operations Research," Beijing: Higher Education Press, 1993.

[4] Wei shixiao, "Weapon Systems Engineering," Beijing: National Defence Industry Press, 1989.

[5] Xu weixin, qin yinxiao, "Reliability Engineering," Beijing: Air Force Logistics Press, 1987.

[6] Wang rongxin, "Stochastic process," Xi'an Jiaotong University Press, 1987.

[7] Liu ming, liu peng, "Weaponry demonstration method and application development system," Beijing: National Defense University Press, 2000.

[8] Zhang jian. Systematic analysis of the effectiveness of military equipment optimization and simulation. Beijing: National Defense University Press, 2000.

[9] Guo kai, Research on Operational Effectiveness firearms comprehensive assessment methods Tactical Missile Technology, 2006.

[10]Wang kai, zhao dingkai, Weaponry Operational Test Beijing: National Defense University Press, 2012. 